\title{
Endoplasmic reticulum stress in the pathogenesis of fibrotic disease
}

\author{
Jonathan A. Kropski ${ }^{1,2}$ and Timothy S. Blackwell ${ }^{1,2,3}$ \\ 'Division of Allergy, Pulmonary and Critical Care Medicine, Department of Medicine, Vanderbilt University Medical Center, Nashville, Tennessee, USA. ²Department of Veterans Affairs Medical Center, \\ Nashville, Tennessee, USA. ${ }^{3}$ Department of Cell and Developmental Biology, Vanderbilt University School of Medicine, Nashville, Tennessee, USA.
}

\begin{abstract}
Eukaryotic cells contain an elegant protein quality control system that is crucial in maintaining cellular homeostasis; however, dysfunction of this system results in endoplasmic reticulum (ER) stress and activation of the unfolded protein response (UPR). Severe or prolonged ER stress is associated with the development of degenerative and fibrotic disorders in multiple organs, as evidenced by the identification of disease-causing mutations in epithelial-restricted genes that lead to protein misfolding or mistrafficking in familial fibrotic diseases. Emerging evidence implicates ER stress and UPR signaling in a variety of profibrotic mechanisms in individual cell types. In epithelial cells, ER stress can induce apoptosis, inflammatory signaling, and epithelial-mesenchymal transition. In other cell types, ER stress is linked to myofibroblast activation, macrophage polarization, and T cell differentiation. ER stress-targeted therapies have begun to emerge using approaches that range from global enhancement of chaperone function to selective targeting of activated ER stress sensors and other downstream mediators. As the complex regulatory mechanisms of this system are further clarified, there are opportunities to develop new disease-modifying therapeutic strategies in a wide range of chronic fibrotic diseases.
\end{abstract}

\section{Introduction}

Tissue fibrosis is a pathologic hallmark of many chronic diseases (1). Progressive architectural remodeling characterized by extensive production of collagen and extracellular matrix commonly accompanies organ dysfunction and failure. Acute or insidious injury almost invariably precedes the development of tissue fibrosis (2). Diverse antecedent events, including tissue ischemia, infection/inflammation, and toxic exposures, can lead to fibrotic remodeling, suggesting that convergent molecular mechanisms culminate in the pathology of tissue fibrosis (1). There is great interest in defining these molecular mechanisms and identifying novel therapeutic targets for a wide range of chronic fibrotic diseases (3).

Abnormalities in protein folding and quality control are cardinal features of aging (4) and have been detected in many chronic degenerative and fibrotic disorders. Initial indications that the protein quality control system may play a direct role in tissue fibrosis emerged from studies of Mendelian forms of fibrotic disease, including familial interstitial pneumonia (the familial form of idiopathic pulmonary fibrosis [IPF]) (5), familial forms of chronic kidney disease (6), and $\alpha_{1}$-antitrypsin-related ( $\alpha 1 \mathrm{AT}$-related) cirrhosis (7). In each case, germline mutations were identified that result in defects in folding and/or processing of a nascent peptide, leading to induction of endoplasmic reticulum (ER) stress and activation of a signaling network known as the unfolded protein response (UPR). As described in more detail below, subsequent work has demonstrated UPR activation in progressive fibrotic diseases involving different organs.

Conflict of interest: The authors have declared that no conflict of interest exists. Reference information: J Clin Invest. 2018;128(1):64-73.

https://doi.org/10.1172/JCI93560.

\section{The ER quality control system}

The ER contains an elegant system designed to facilitate the proper folding and trafficking of proteins, particularly those destined for secretory pathways. Within the ER, a variety of chaperone proteins are involved in protein folding and trafficking. In physiologic states of rapid cellular proliferation (for example, malignancy) or in highly secretory cells (plasma cells, pancreatic $\beta$ cells, and alveolar type II epithelial cells), the activation of a wellcoordinated series of transcriptional and translational changes promotes homeostasis. UPR signaling is mediated through three effector pathways that involve activation of PKR-like ER kinase (PERK), activating transcription factor 6 (ATF6), and inositolrequiring enzyme $1 \alpha$ (IRE1 $\alpha$ ). Activation of UPR signaling can modulate new protein synthesis, increase production of ER chaperones to improve protein folding, and induce components of the ER-associated degradation (ERAD) system.

The immunoglobulin heavy chain chaperone protein Bip (also known as glucose-related peptide 78, GRP78) is a heat-shock protein family member that is central to UPR regulation. Under normal conditions in the ER, Bip is constitutively bound to the three ER sensors (PERK, ATF6, and IRE1 $\alpha$ ) and suppresses their signaling (2). Bip also binds to misfolded proteins in the ER, and thus, as misfolded proteins accumulate, Bip binding to the three UPR sensors is reduced $(3,4)$.

PERK undergoes dimerization and autophosphorylation upon dissociation from Bip, which in turn leads to phosphorylation of eukaryotic translation initiation factor $2 \alpha($ EIF $2 \alpha)$ at Ser51, resulting in a global reduction in mRNA translation (8). However, eIF $2 \alpha$ phosphorylation can also increase translation of selected mRNAs, including activating transcription factor 4 (ATF4) (9). ATF4 then acts to increase expression of ATF3, which in turn promotes expression of genes related to antioxidant responses, amino acid synthesis, and autophagy (13). 


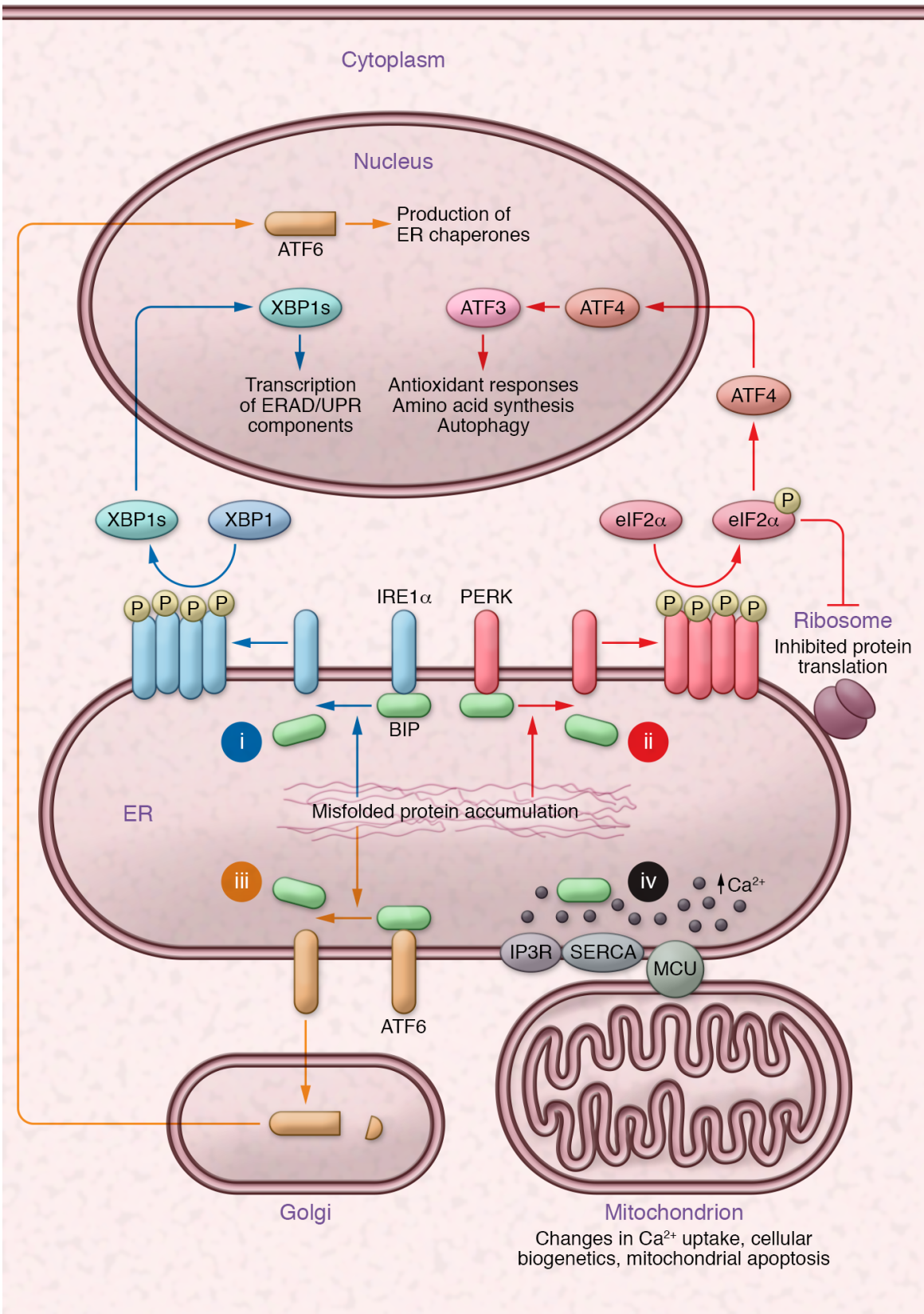

Figure 1. Overview of ER stress-related signaling. Bip binds to accumulating misfolded proteins in the ER, leading to its dissociation from the three ER stress sensors, IRE1 $\alpha$, PERK, and ATF6. (i) Dissociation from Bip allows IRE1 $\alpha$ to multimerize and autophosphorylate, activating endoribonuclease activity that leads to alternative splicing of the transcription factor XBP1. Spliced XBP1 (XBP1s) then translocates to the nucleus and promotes transcription of components of the ERAD system. Oligomerized IRE1 $\alpha$ loses stringency of endoribonuclease activity and activates regulated IRE1-dependent decay (RIDD), thereby degrading mRNA and miRNAs. (ii) Bip dissociation leads to dimerization and autophosphorylation of PERK, which phosphorylates elF $2 \alpha$ to inhibit protein translation and signals for ATF4 nuclear translocation. Once in the nucleus, ATF4 activates ATF3, which induces adaptive antioxidant responses, promotes amino acid synthesis, and promotes autophagy. (iii) Bip dissociation from ATF6 permits its transit from the ER to the Golgi, where further processing allows trafficking to the nucleus and subsequent increases in production of ER chaperones. (iv) Bip and other ER chaperones serve as calcium-binding proteins. The ER tightly controls the cytosolic calcium pool available for mitochondrial uptake through the mitochondrial calcium uniporter (MCU) via sarcoendoplasmic reticulum $\mathrm{Ca}^{2+}$ ATPase (SERCA) and the inositol triphosphate receptor (IP3R). Through its regulation of calcium flux, the ER plays a central role in the regulation of cellular bioenergetics and mitochondrial mechanisms of apoptosis.

Following its release from Bip, ATF6 is trafficked to the Golgi apparatus, where it undergoes a cleavage event that releases the cytosolic domain. This activated form of ATF6 enters the nucleus and acts to enhance transcription of target genes, including the ER chaperones Bip, GRP94, calreticulin, and components of the ERAD system (14-16).

Activated IRE1 $\alpha$ possesses endoribonuclease activity, which leads to selective removal of 26 base pairs from the mRNA encoding transcription factor X-box protein-1 (XBP1), producing a transcriptionally active form (XBP1s). XBP1s then migrates to the nucleus, where it promotes transcription of components of the ERAD system, including ER degradation-enhancing $\alpha$-mannosidase-like protein (EDEM). Activated IRE1 $\alpha$ can either homodimerize or oligomerize and autophosphorylate. Oligomerized polyphosphorylated IRE1 $\alpha$ appears to have less strin- gent mRNA endonuclease activity and acts to promote generalized degradation of mRNAs, as well as microRNAs (miRNAs), through a process known as IRE1-dependent decay (RIDD) (17).

These three arms of UPR signaling work in concert to maintain cellular homeostasis in the setting of ER stress (Figure 1). However, under severe or prolonged ER stress, this process may become maladaptive and promote cellular dysfunction and death.

\section{Profibrotic cellular phenotypes associated with ER stress}

Although the mechanisms connecting ER stress with fibrosis have been challenging to unravel, most studies to date have focused on proapoptotic (18) or proinflammatory effects of ER stress. In addition, direct profibrotic effects related to mediator production and acquisition of mesenchymal characteristics (epithelial-mesenchymal 


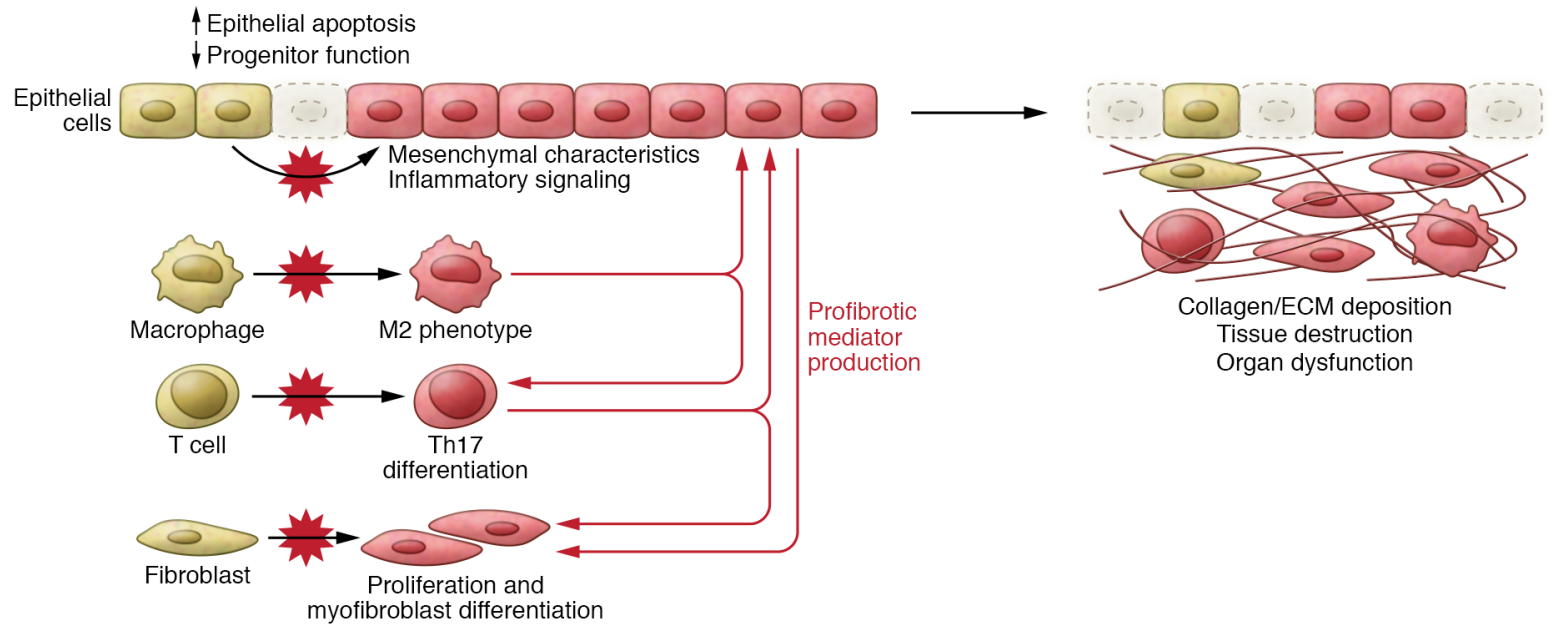

Figure 2. Mechanisms of fibrosis related to ER stress. In epithelial cells, ER stress induces a profibrotic microenvironment by promoting apoptosis, suppressing progenitor cell function, activating inflammatory signaling pathways, and inducing production of profibrotic mediators that promote fibroblast proliferation and myofibroblast differentiation. ER stress signaling in T lymphocytes suppresses Th1 and Th2 polarization and drives Th17 polarization, which can promote fibrosis through interactions with epithelium and fibroblasts. In macrophages, ER stress facilitates acquisition of the M2 phenotype, which is accompanied by enhanced production of profibrotic mediators.

transition) $(19,20)$ may also contribute to fibrotic remodeling in a context-specific manner.

Apoptosis. Apoptosis of epithelial cells in the lungs and other organs is linked to fibrotic remodeling, presumably by inhibiting re-epithelialization after injury or by impairing barrier functions of the epithelium. The ER is intricately associated with mitochondria through a large number of mitochondria-associated membranes (reviewed in ref. 21) and plays a critical role in regulating cellular bioenergetics and cell-death signaling through its sequestration of intracellular calcium. Uptake of calcium by the mitochondria through the low-affinity mitochondrial calcium uniporter complex (MCU) is crucial for maintaining the mitochondrial membrane potential $(22,23)$; the availability of cytosolic calcium for uptake through the MCU is highly regulated by the ER membrane-resident sarco/endoplasmic reticulum $\mathrm{Ca}^{2+} /$ ATPase (SERCA) pump, ryanodine receptors, and inositol 1,4,5-triphosphate receptors (IP3Rs) (21). Numerous ER-resident chaperones, including Bip and calreticulin, function as calciumbinding proteins (21), and impairment of SERCA function increases calcium leakage, promotes ER stress, and enhances apoptotic susceptibility $(24,25)$. Impairment of IP3R-dependent calcium transfer also leads to reduced mitochondrial ATP production and promotes autophagy (26).

In addition to direct ER-mitochondrial interactions, ER stress can initiate proapoptotic signaling through each of the three arms of the UPR cascade. ATF4 induction downstream of PERK can activate C/EBP homologous protein (CHOP), a well-studied inducer of apoptosis (reviewed in ref. 27). IRE1 $\alpha$ and ATF6 pathways can also contribute to CHOP induction in some settings (28). $\mathrm{CHOP}$ is a bifunctional transcription factor that can promote cell death by influencing expression of pro- and antiapoptotic factors, including BCL-2 and BH3-only family members (29). IRE1 $\alpha$ phosphorylation also leads to activation of c-Jun $\mathrm{NH}_{2}$-terminal kinase (JNK), which can promote apoptosis as well as increase RIDD, which can have proapoptotic effects. Also, caspase-4 (and its murine homolog, caspase-12) is an ER membrane-resident caspase that becomes activated in the setting of ER stress (30) and induces apoptosis through activation of caspase-9 (14). The precise mechanism of ER stress-induced caspase-4 activation remains uncertain, but it may be mediated through calcium signaling (32).

Inflammatory signaling. Persistent inflammation can result in tissue injury and aberrant repair that facilitates fibrotic remodeling of affected tissues. The UPR has been shown to activate proinflammatory transcription factors such as NF- $\mathrm{BB}$ and AP-1 (33) through IRE1 $\alpha$-mediated induction of TRAF2 signaling (17). TRAF2 can activate NF- $\kappa$ B in a NOD1/2- and RIP1-dependent cascade (18) and can activate AP-1 through JNK phosphorylation (17). PERK activation can also result in increased NF- $\kappa B$ activity through translational suppression of $\mathrm{I} \kappa \mathrm{B} \alpha$ (36). However, activation of inflammatory signaling appears to be context and cell type dependent, as NF- $\kappa \mathrm{B}$ activation is not observed in all models of ER stress $(20,21)$. Suppression of TNF- $\alpha$ and LPS-induced NF- $\kappa B$ activation (22), as well as inhibition of NF- $\mathrm{kB}$-driven cytokine production, has even been reported in some systems (23).

In addition to transcriptional effects on inflammatory pathways, ER stress can alter the phenotype of immune/inflammatory cells, particularly macrophages. NF- $\kappa \mathrm{B}$ activation supports production of a variety of mediators associated with classically activated (M1) macrophages (41). In addition, ER stress has also been linked to activation of NLRP3 inflammasomes (42), which process the M1-associated cytokines IL-1 $\beta$ and IL-18 for secretion. A recent study showed that genetic deletion of IRE1 $\alpha$ in macrophages limited M1 polarization and increased the alternatively activated (M2) macrophage phenotype (43). Similarly, CHOP deficiency was shown to prevent M1 macrophage polarization in a model of high-fat diet-induced obesity (44). In contrast, however, genetic deletion of CHOP reportedly decreased M2 macrophage polarization in a model of pulmonary fibrosis (45), and ex vivo M2 polarization of mouse peritoneal macrophages has been shown to depend on ER stress-induced JNK activation (46). 
In addition to innate immunity, UPR signaling plays a role in the adaptive immune system. Plasma cell and dendritic cell maturation and differentiation requires ER stress-induced activation of IRE1 $\alpha(47,48)$. ER stress may also play a role in regulating $\mathrm{T}$ cell responses, in particular, polarization of Th17 cells (49), which have been implicated in lung and skin fibrosis $(50,51)$. Induction of ER stress by hypoxia or nutrient deprivation promotes Th17 cell differentiation independent of TGF- $\beta$, which is mediated at least in part through XBP1 (49).

Thus, available evidence indicates that ER stress modulates inflammatory signaling and immune/inflammatory cell phenotypes in complex ways that depend on the cell type and stimulus.

Epithelial-mesenchymal signaling and differentiation. In epithelial cells, induction of ER stress has been shown to cause a phenotypic shift characterized by adoption of mesenchymal cell-like morphology and reduced expression of epithelial markers. This phenotype shift includes induction of vimentin, $\mathrm{N}$-cadherin, and $\alpha$-smooth muscle actin ( $\alpha \mathrm{SMA})$, markers typically associated with mesenchymal cells $(33,34)$. Acquisition of mesenchymal characteristics may allow these cells to more directly contribute to wound repair and tissue remodeling. In addition, recent work indicates that ER stress can suppress Wnt-driven epithelial stem/progenitor cell function downstream of $\beta$-catenin nuclear localization (52), suggesting that ER stress may inhibit the self-renewal capacity of local progenitor cell niches.

UPR activation has been shown to alter the function and activation of fibroblasts (53), the cell type primarily responsible for collagen and matrix deposition. UPR activation promotes TGF- $\beta$ mediated myofibroblast differentiation $(54,55)$ at least in part through IRE1 $\alpha$-mediated regulation of miRNAs (56). For example, activated IRE1 $\alpha$ can degrade miR-150, which negatively regulates aSMA expression (57).

Together, available data indicate that ER stress promotes fibrosis by enhancing apoptosis of epithelial cells, promoting profibrotic cytokine production from epithelial and immune cells, and enhancing activation of myofibroblasts (Figure 2).

\section{Mechanisms of ER stress induction in fibrotic diseases}

The case for a mechanistic relationship between ER stress and fibrosis is bolstered by the identification of rare disease-causing mutations that result in expression of misfolded proteins. In the lung, mutations in the genes encoding surfactant protein $\mathrm{C}$ (SFTPC) (58-60) and surfactant protein A2 (SFTPA2) (61), which result in ER stress in type II alveolar epithelial cells, have been identified in families with IPF. In the kidney, nephrin (62), laminin $\beta 2$ (63), and podocin (64) mutations that result in ER stress in podocytes have been identified in families with chronic kidney disease. In the liver, Z-allele $\alpha 1 \mathrm{AT}$ produces a misfolded protein product in hepatocytes that accumulates in the ER and is associated with development of cirrhosis in some individuals homozygous for the mutant allele (65). Each of these disease-causing genetic variants shares the common feature of producing highly expressed protein products that are misfolded and mistrafficked, resulting in ER stress in epithelial cells of the target organ.

In addition to expression of defective proteins, expression of exogenous viral proteins can cause ER stress and fibrotic re- modeling in target organs. Hepatitis $\mathrm{C}$ virus $(\mathrm{HCV})$ infection is associated with ER stress in hepatocytes $(66,67)$. Before the development of effective antiviral therapies, HCV infection frequently progressed to cirrhosis and end-stage liver disease. In the lung, chronic infection of alveolar epithelial cells with human herpesviruses has been associated with ER stress and development of IPF (60).

Despite the examples given above, the proximate cause of ER stress in fibrotic diseases is often not apparent; however, numerous metabolic and bioenergetic stressors are known to cause ER stress and activate the UPR in vitro. Cellular aging has also been associated with reduced ER capacity and increased susceptibility to ER stress, likely mediated at least in part through mitochondrial dysfunction $(68,69)$. In addition, oxidative stress $(70)$, altered calcium homeostasis (71), and cellular hypoxia (72-75) can impair protein folding and processing, culminating in ER stress.

\section{ER stress in chronic fibrotic disorders}

Pulmonary fibrosis. IPF is a progressive form of tissue fibrosis that typically leads to respiratory failure within 3-5 years of diagnosis (76). Familial clustering of IPF, known as familial interstitial pneumonia (FIP), has been observed since the 1950s (77). In 2002, the first genetic cause of FIP was identified in a large family that included IPF patients and individuals with childhood onset of interstitial lung disease (78). Affected individuals shared a rare missense variant (L188Q) in the gene encoding surfactant protein $\mathrm{C}(\mathrm{SP}-\mathrm{C})$, which is expressed exclusively in type II alveolar epithelial cells. This variant is adjacent to a cysteine residue in pro-SP-C that is required for proper folding (79). After studies showed that misfolded pro-SP-C aggregates in the ER and activates the UPR $(60,80)$, additional studies from several groups demonstrated that other disease-associated mutations in the C-terminal portion (or BRICHOS domain) of pro-SP-C result in expression of a misfolded protein $(20,38,58,59,80)$. In addition to heterozygous SFTPC mutations, heterozygous mutations in another surfactant protein gene, SFTPA2, have been linked to aberrant protein processing, ER stress, and pulmonary fibrosis in families (61).

The identification of ER stress in alveolar epithelial cells expressing mutant surfactant proteins prompted studies in sporadic IPF patients, which identified ER stress as a common feature in IPF lungs (60). Increased expression of a number of ER stress markers, including Bip, XBP1, ATF4, ATF6, and CHOP, has been reported in IPF patient samples, primarily localized to hyperplastic type II alveolar epithelial cells in areas of fibrotic remodeling $(60,68,81,82)$. In addition, some asymptomatic first-degree relatives of FIP patients have evidence of ER stress in alveolar epithelial cells (83), suggesting that ER stress could be an early driver of disease pathogenesis. While these studies provide compelling evidence of ER stress in the IPF lung, the etiology of ER stress is uncertain. In vitro and preclinical animal models indicate that cigarette smoke (84-86), asbestos (87), and environmental particulates (88) can induce ER stress in alveolar epithelial cells. In addition, the colocalization of ER stress markers with herpesvirus antigens in the alveolar epithelium of IPF patients (60) suggests that viral protein expression could be a factor.

Animal models have helped to clarify a role for ER stress in development of pulmonary fibrosis. Constitutive expression of 
a mutant form of SFTPC or overexpression of the mature SP-C peptide disrupts lung development and results in perinatal mortality with disrupted lung development, accumulation of protein in the ER, and evidence of ER stress $(58,89)$. We used an inducible transgenic model to overexpress the L188Q mutant form of human SFTPC in alveolar epithelial cells using the murine SP-C promoter (20). Following doxycycline treatment, the transgene localized to the ER and activated the UPR, but no evidence of lung fibrosis was found even with long-term transgene expression. However, following treatment with low-dose bleomycin, lung fibrosis and alveolar epithelial cell apoptosis were markedly exacerbated in animals expressing mutant SFTPC. Similarly, intratracheal treatment with the ER stress-inducing agent tunicamycin failed to spontaneously cause fibrosis, but worsened bleomycin-induced lung fibrosis. Together, these studies indicate that induction of ER stress in lung is not sufficient to cause lung fibrosis; rather, ER stress exacerbates the response to fibrogenic stimuli.

Although epithelial cell apoptosis is implicated as an important factor in determining the development and progression of lung fibrosis, the relevant UPR pathway(s) and effector molecules that determine epithelial cell death and survival in the presence of fibrotic stimuli remain uncertain. For example, the role of $\mathrm{CHOP}$ has been investigated with conflicting results. In two reports, CHOP-deficient mice exhibited comparative reductions in hydroxyproline content and histologic fibrosis after bleomycin treatment $(45,90)$. In contrast, another group reported markedly worse survival and increased fibrosis in CHOP-deficient mice following bleomycin treatment (91). The latter study also showed that mice with heterozygous loss of Bip (which would be expected to exacerbate ER stress) were protected from lung fibrosis via increased CHOP-dependent macrophage apoptosis.

IPF is a disease that occurs with aging (median onset at age 65), and altered proteostasis is a hallmark of aging. One study using murine herpesvirus 68 infection found that ER stress in the lung was substantially greater in old mice after infection, and this correlated with development of lung fibrosis (69). While the reason for increased susceptibility to ER stress in aging is not well understood, an important clue may be the finding that expression of the mitochondrial protective factor PINK1 is reduced in aging and IPF lungs (68). ER stress was shown to downregulate PINK1 in mitochondria, thus altering bioenergetics in affected epithelium and promoting apoptosis. In turn, loss of PINK1 can induce ER stress (92), potentially leading to a feedback loop with persistent ER stress that facilitates fibrotic remodeling.

Available data indicate that ER stress contributes to a vulnerable epithelial state in IPF, resulting in increased susceptibility to apoptotic stimuli and impaired epithelial regeneration following injury. However, the impact of ER stress on disease pathogenesis remains incompletely understood, and more information is needed regarding the causes and consequences of ER stress in IPF.

Chronic kidney disease. ER stress related to both genetic and environmental factors has been identified in chronic kidney disease (CKD) $(6,93-98)$. Aberrant gene products associated with mutations in several genes expressed by podocytes, including collagen IV (97), $\alpha$-actinin-4 (99), laminin (63), nephrin (62), and podocin (64), can induce ER stress and UPR activation, which have been shown to contribute to podocyte injury/apoptosis, proteinuria, and CKD. In addition, albumin has been shown to induce ER stress in tubular epithelial cells by altering intracellular calcium levels, resulting in apoptosis via UPR-dependent upregulation of lipocalin 2 (100). Together, these studies implicate ER stress as a factor in both induction of proteinuria and mediation of its toxic effects. In another form of familial CKD, autosomal dominant tubulointerstitial kidney disease, genetic mutations in uromodulin $(98,101,102)$ have been reported to induce ER stress in epithelial cells in the thick ascending limb of the loop of Henle and cause progressive tubulointerstitial fibrosis. A recently published transgenic mouse model with knock-in of a human missense uromodulin mutation showed that ER stress-regulated factors, including tribbles-3, can determine pathology by sensitizing cells to TNF- $\alpha$ - and TRAIL-induced apoptosis (103).

In addition to genetic causes of ER stress, environmental insults such as hypoxia, increased glucose, and drugs (e.g., cyclosporine) can induce ER stress in the kidney $(6,96)$. ER stress is well documented in diabetic kidneys (104-106); however, it is not entirely clear whether it is protective or pathogenic. While diabetic CHOP-deficient mice develop less proteinuria compared with controls (107), podocyte-specific XBP1 deficiency or overexpression of ATF6 worsens diabetic nephropathy (108).

Recent studies have identified a new mechanistic connection between ER stress and kidney disease through the ER-associated protein reticulon 1 (RTN1) (104, 109-112), which is associated with acute kidney injury and progression to CKD in animal models and in humans. Increased expression of RTN1 (particularly the RTN1A isoform) induces apoptosis of renal epithelial cells through ER stress-induced activation of PERK and downstream induction of CHOP (104). Knockdown or inhibition of RTN1A expression attenuated ER stress, apoptosis, and renal injury fibrosis in models of unilateral ureteral obstruction (104), diabetic nephropathy (111), and albumin-overload kidney disease (112). It remains to be seen whether this mechanism of ER stress-dependent injury and remodeling is relevant in other organs.

Another mechanism by which ER stress signaling affects diabetic nephropathy is regulation of long noncoding RNA (lncRNA) (105). In a murine model, a cluster of several dozen miRNAs encoded by a host lncRNA was found to be upregulated in the setting of ER stress, both in glomeruli of diabetic mice and in cultured mesangial cells (105). This miRNA cluster is predicted to regulate a variety of signaling processes relevant to fibrosis, including the TGF- $\beta$ pathway (105). Although more work is needed, it is likely that future studies will identify a larger role for lncRNAs in regulating ER stress-related signaling.

Hepatic fibrosis. ER stress has been observed in several forms of chronic liver disease, including cirrhosis associated with $\mathrm{HCV}$ infection (113) or mutant forms of $\alpha 1 \mathrm{AT}$ (65), nonalcoholic steatohepatitis (NASH) (114), and primary biliary cirrhosis (115). Toxic aggregation of misfolded $\mathrm{Z}$-allele $\alpha 1 \mathrm{AT}$ protein is believed to drive a1AT-related liver disease through pathways involving ER stress, autophagy, and other cellular quality control systems (116-118). In $\mathrm{NASH}$, lipids accumulate in hepatocytes when the influx of fatty acids exceeds the clearance capacity (114). Subsequently, lipid overload results in chronic ER stress that, in turn, increases lipogenesis, drives inflammation, and causes hepatocyte apoptosis $(114,119)$. 
In addition to hepatocytes, hepatic stellate cells (HSCs) are also susceptible to ER stress and may be important for hepatic remodeling. It was recently reported that UPR signaling mediates HSC collagen I secretion through XBP1-dependent induction of transport and Golgi organization 1 (TANGO1), thus stimulating liver fibrosis (120). In addition, ER stress was shown to enhance TGF- $\beta$ signaling as a result of decreasing levels of miR-18a in HSCs (121). Further, several recent reports have indicated that upregulation of the growth factor FGF21 may hold promise in preventing ER stress-mediated steatosis (122-125). Despite evidence that ER stress in HSCs contributes to collagen deposition, studies using chemical chaperones 4-phenylbutyric acid or tauroursodeoxycholic acid to reduce ER stress in mice with methionine- and choline-deficient diet-induced hepatic steatosis have reported mixed results regarding disease progression $(126,127)$. Underscoring the complexity of this phenotype, it has also been suggested that ER stress-dependent apoptosis of HSCs could be beneficial in limiting fibrotic remodeling in the liver (119).

A number of studies have suggested that CHOP is an important effector molecule through which ER stress impacts liver fibrosis. Following bile duct ligation, liver fibrosis and acute liver injury are greatly attenuated in CHOP-deficient mice (128). Knockdown of CHOP also protects primary hepatocytes from apoptosis following fatty acid-induced ER stress $(129,130)$. Similarly, hepatocytes from CHOP-deficient mice display reduced apoptosis when exposed to toxic stimuli such as glycochenodeoxycholic acid (128) or intragastric ethanol feeding (131).

ER stress in other chronic fibrotic disorders. Available evidence links ER stress to fibrotic conditions in a variety or organs. For example, ER stress-regulated chronic inflammation drives fibrotic remodeling in inflammatory bowel disease (IBD). In biopsies from IBD patients, increased levels of Bip and spliced XBP1 have been reported, including in segments of the mucosa relatively devoid of inflammation (132). Mice deficient in IRE1 $\beta$ or XBP1 in the intestinal epithelium develop spontaneous gut inflammation and display enhanced proapoptotic signaling in Paneth cells (132). In contrast, however, inducing ER stress by expression of a mutant mucin 2 (Muc2) was shown to cause spontaneous colonic inflammation and an increase in Th1 cytokines (133).

In heart failure, several experimental models have shown induction of Bip, CHOP, and other ER stress markers in cardiomyocytes, along with attenuated fibrosis after treatment with a pharmacologic ER chaperone $(134,135)$. In the skin, interrupting IRE1 $\alpha$ signaling can prevent or reverse myofibroblast activation in cells from patients with systemic sclerosis (57).

\section{ER stress as a therapeutic target in fibrosis}

There is emerging interest in components of the ER stress response as therapeutic targets in the fields of cancer biology, neurodegenerative diseases, and fibrosis. Targeting these pathways presents unique challenges, as current evidence suggests that conventional approaches attempting to block or broadly inhibit signaling through one or more arms of the ER stress pathway may have significant toxicities due to the role of the UPR in cellular homeostasis (33). For example, deletion of XBP1 in lymphoid cells leads to failure of plasma cell differentiation and profound suppression of antibody production (47), which could predispose to infectious complications.
As an alternative, there has long been interest in enhancing protein chaperone function in the ER. In support of this approach, treatment with pharmacologic chaperones such as sodium phenylbutyrate has been shown to reduce ER stress and reduce or prevent disease in a variety of preclinical disease models (136-140). Other creative strategies to "fine-tune" ER stress signaling may also hold promise. In one intriguing report, a small-molecule allosteric modulator was shown to selectively inhibit oligomerized IRE1 $\alpha$ but permit signaling through dimerized complexes (141), suggesting that it may be possible to specifically target pathologic signaling through this molecule. Leveraging alternative cellular quality control mechanisms such as autophagy may also alleviate pathologic ER stress in certain circumstances (116).

\section{The future: ER stress and the microenvironment}

Studies to date have provided remarkable insights into the molecular events that mediate ER stress within a cell; however, the effects of pathologic ER stress in a given microenvironment remain incompletely understood. Given the dueling homeostatic and pathologic functions of the ER stress machinery, targeting the downstream consequences of ER stress may be the most practical and promising therapeutic approach. However, emerging clues suggesting non-cell-autonomous effects of ER stress suggest that a broader context may be required for considering ER stress-targeted therapies (142). For example, transfer of supernatant from mucopurulent material from cystic fibrosis lungs can induce ER stress in normal human bronchial epithelial cells (143). Similarly, conditioned medium from prostate cancer cells was shown to induce ER stress in cultured cells (144). The mediators of this effect have not yet been fully elucidated, but may involve TLR4 signaling (144). In Caenorhabditis elegans, neuron-specific expression of spliced XBP1 induced ER stress in adjacent non-neuronal tissue, suggesting that paracrine communications may play a role in ER stress induction (145). Further work is needed to clarify the mechanisms of this "transmissible ER stress." Intriguingly, a recent report indicates that multivesicular body formation and exosome release are increased in the setting of ER stress (146), suggesting that the cargo of ER stress-derived exosomes could contribute to cell-cell interactions in fibrotic tissue. Technological advances in single-cell analytics should allow rapid growth in our understanding of the role of intracellular communications in disease pathology and are likely to uncover downstream mediators that are promising therapeutic targets.

\section{Conclusion}

It has been more than two decades since the earliest descriptions of the cellular consequences of ER stress, and there have been significant advances in understanding homeostatic and pathologic signaling through this pathway. The best-studied disease mechanism (ER stress-induced apoptosis) may have high relevance to chronic neurodegenerative diseases and cancer therapy (28), but its contribution to chronic fibrotic disorders is less certain. It appears unlikely that enhanced cell death and/or turnover is sufficient to explain the striking pathologic changes observed in lung, 
kidney, liver, and other forms of tissue fibrosis related to ER stress. The challenge for the coming years is to better elucidate how the cellular phenotype of ER stress culminates in chronic fibrotic diseases, and how to leverage these complex signaling mechanisms to promote adaptive tissue repair and homeostasis.

\section{Acknowledgments}

This work was supported by NIH National Heart, Lung, and Blood Institute grants K08HL130595 (to JAK), P01HL92870 (to TSB), and R01HL085317 (to TSB); the Department of Veterans Affairs (to TSB); the Francis Family Foundation (to JAK); and the Pulmonary Fibrosis Foundation (to JAK).

Address correspondence to: Jonathan A. Kropski or Timothy S. Blackwell, Division of Allergy, Pulmonary and Critical Care Medicine, Department of Medicine, Vanderbilt University School of Medicine, 1611 21st Avenue South, T-1218 Medical Center North, Nashville, Tennessee 37232, USA. Phone: 615.322.3412; E-mail: jon.kropski@ vanderbilt.edu (J.A. Kropski), timothy.blackwell@vanderbilt.edu (T.S. Blackwell).
1. Rockey DC, Bell PD, Hill JA. Fibrosis--a common pathway to organ injury and failure. $N$ EnglJ Med. 2015;372(12):1138-49.

2. Wynn TA. Common and unique mechanisms regulate fibrosis in various fibroproliferative diseases. JClin Invest. 2007;117(3):524-9.

3. Nanthakumar CB, Hatley RJD, Lemma S, Gauldie J, Marshall RP, Macdonald SJF. Dissecting fibrosis: therapeutic insights from the small-molecule toolbox. Nat Rev Drug Discov. 2015;14(10):693-720.

4. López-Otín C, Blasco MA, Partridge L, Serrano M, Kroemer G. The hallmarks of aging. Cell. 2013;153(6):1194-217.

5. Kropski JA, Lawson WE, Young LR, Blackwell TS. Genetic studies provide clues on the pathogenesis of idiopathic pulmonary fibrosis. Dis Model Mech. 2013;6(1):9-17.

6. Taniguchi M, Yoshida H. Endoplasmic reticulum stress in kidney function and disease. Current Opin Nephrol Hypertens. 2015;24(4):345-50.

7. Marciniak SJ, et al. New concepts in alpha-1 antitrypsin deficiency disease mechanisms. Ann Am Thorac Soc. 2016;13 Suppl 4:S289-S296.

8. Bertolotti A, Zhang Y, Hendershot LM, Harding HP, Ron D. Dynamic interaction of BiP and ER stress transducers in the unfolded-protein response. Nat Cell Biol. 2000;2(6):326-332.

9. Gardner BM, Walter P. Unfolded proteins are Ire1-activating ligands that directly induce the unfolded protein response. Science. 2011;333(6051):1891-1894.

10. Carrara M, Prischi F, Nowak PR, Kopp MC, Ali MM. Noncanonical binding of BiP ATPase domain to Ire1 and Perk is dissociated by unfolded protein $\mathrm{CH} 1$ to initiate $\mathrm{ER}$ stress signaling. eLife. 2015;4:e03522.

11. Krishnamoorthy T, Pavitt GD, Zhang F, Dever TE, Hinnebusch AG. Tight binding of the phosphorylated alpha subunit of initiation factor 2 (eIF2alpha) to the regulatory subunits of guanine nucleotide exchange factor eIF2B is required for inhibition of translation initiation. Mol Cell Biol. 2001;21(15):5018-5030.

12. Harding HP, et al. Regulated translation initiation controls stress-induced gene expression in mammalian cells. Mol Cell. 2000;6(5):1099-1108.

13. Zhao J, Li X, Guo M, Yu J, Yan C. The common stress responsive transcription factor ATF3 binds genomic sites enriched with $\mathrm{p} 300$ and H3K27ac for transcriptional regulation. BMC Genomics. 2016;17(1):335.

14. Ye J, et al. ER stress induces cleavage of membranebound ATF 6 by the same proteases that process SREBPs. Mol Cell. 2000;6(6):1355-1364.
15. Lee K, et al. IRE1-mediated unconventional mRNA splicing and S2P-mediated ATF6 cleavage merge to regulate XBP1 in signaling the unfolded protein response. Genes Dev. 2002;16(4):452-466.

16. Yoshida H, Matsui T, Yamamoto A, Okada T, Mori K. XBP1 mRNA is induced by ATF6 and spliced by IRE1 in response to ER stress to produce a highly active transcription factor. Cell. 2001;107(7):881-891.

17. Hollien J, Lin JH, Li H, Stevens N, Walter P, Weissman JS. Regulated Ire1-dependent decay of messenger RNAs in mammalian cells. J Cell Biol. 2009;186(3):323-331.

18. Tanjore H, Blackwell TS, Lawson WE. Emerging evidence for endoplasmic reticulum stress in the pathogenesis of idiopathic pulmonary fibrosis. Am J Physiol Lung Cell Mol Physiol. 2012;302(8):L721-L729.

19. Tanjore H, et al. Alveolar epithelial cells undergo epithelial-to-mesenchymal transition in response to endoplasmic reticulum stress. J Biol Chem. 2011;286(35):30972-30980.

20. Zhong Q, et al. Role of endoplasmic reticulum stress in epithelial-mesenchymal transition of alveolar epithelial cells: effects of misfolded surfactant protein. Am J Respir Cell Mol Biol. 2011;45(3):498-509.

21. Marchi S, et al. Mitochondrial and endoplasmic reticulum calcium homeostasis and cell death [published online ahead of print May 5, 2017]. Cell Calcium. https://doi.org/10.1016/j. ceca.2017.05.003.

22. Drago I, Pizzo P, Pozzan T. After half a century mitochondrial calcium in- and efflux machineries reveal themselves. Embo J. 2011;30(20):4119-4125.

23. Williams GS, Boyman L, Lederer WJ. Mitochondrial calcium and the regulation of metabolism in the heart. J Mol Cell Cardiol. 2015;78:35-45.

24. Chami M, et al. SERCA1 truncated proteins unable to pump calcium reduce the endoplasmic reticulum calcium concentration and induce apoptosis. J Cell Biol. 2001;153(6):1301-1314.

25. Chami M, Oules B, Szabadkai G, Tacine R, Rizzuto R, Paterlini-Brechot P. Role of SERCA1 truncated isoform in the proapoptotic calcium transfer from ER to mitochondria during ER stress. Mol Cell. 2008;32(5):641-651.

26. Cardenas C, et al. Essential regulation of cell bioenergetics by constitutive InsP3 receptor $\mathrm{Ca}^{2+}$ transfer to mitochondria. Cell. 2010;142(2):270-283.

27. Hetz C, Saxena S. ER stress and the unfolded protein response in neurodegeneration. Nat Rev Neurol. 2017;13(8):477-491.

28. Kim I, Xu W, Reed JC. Cell death and endoplas- mic reticulum stress: disease relevance and therapeutic opportunities. Nat Rev Drug Discov. 2008;7(12):1013-1030

29. Pihan P, Carreras-Sureda A, Hetz C. BCL-2 family: integrating stress responses at the ER to control cell demise. Cell Death Differ. 2017;24(9):1478-1487.

30. Morishima N, Nakanishi K, Takenouchi H, Shibata T, Yasuhiko Y. An endoplasmic reticulum stress-specific caspase cascade in apoptosis. J Biol Chem. 2002;277(37):34287-34294.

31. Nakagawa T, et al. Caspase-12 mediates endoplasmic-reticulum-specific apoptosis and cytotoxicity by amyloid-beta. Nature. 2000;403(6765):98-103.

32. Casson CN, et al. Human caspase- 4 mediates noncanonical inflammasome activation against gram-negative bacterial pathogens. Proc Natl Acad Sci U S A. 2015;112(21):6688-6693.

33. Pahl HL, Baeuerle PA. A novel signal transduction pathway from the endoplasmic reticulum to the nucleus is mediated by transcription factor NF-kappa B. EMBO J. 1995;14(11):2580-2588.

34. Kaneko M, Niinuma Y, Nomura Y. Activation signa of nuclear factor-kappa B in response to endoplasmic reticulum stress is transduced via IRE1 and tumor necrosis factor receptor-associated factor 2. Biol Pharm Bull. 2003;26(7):931-935.

35. Keestra-Gounder AM, et al. NOD1 and NOD2 signalling links ER stress with inflammation. Nature. 2016;532(7599):394-397.

36. Deng J, et al. Translational repression mediates activation of nuclear factor kappa B by phosphorylated translation initiation factor 2. Mol Cell Biol. 2004;24(23):10161-10168.

37. Lawson WE, et al. Endoplasmic reticulum stress enhances fibrotic remodeling in the lungs. Proc Natl Acad Sci U S A. 2011;108(26):10562-10567.

38. Maguire JA, Mulugeta S, Beers MF. Endoplasmic reticulum stress induced by surfactant protein $C$ BRICHOS mutants promotes proinflammatory signaling by epithelial cells. Am J Respir Cell Mol Biol. 2011;44(3):404-414.

39. Hayakawa K, et al. ER stress depresses NF-kappaB activation in mesangial cells through preferential induction of C/EBP beta. J Am Soc Nephrol. 2010;21(1):73-81.

40. Komura T, Sakai Y, Honda M, Takamura T, Wada T, Kaneko S. ER stress induced impaired TLR signaling and macrophage differentiation of human monocytes. Cell Immunol. 2013;282(1):44-52.

41. Blackwell TS, Christman JW. The role of nuclear factor-kappa B in cytokine gene regulation. Am J Respir Cell Mol Biol. 1997;17(1):3-9. 
42. Lebeaupin C, et al. ER stress induces NLRP3 inflammasome activation and hepatocyte death. Cell Death Dis. 2015;6:e1879.

43. Shan B, et al. The metabolic ER stress sensor IRE1alpha suppresses alternative activation of macrophages and impairs energy expenditure in obesity. Nat Immunol. 2017;18(5):519-529.

44. Grant R, Nguyen KY, Ravussin A, Albarado D, Youm YH, Dixit VD. Inactivation of C/ ebp homologous protein-driven immunemetabolic interactions exacerbate obesity and adipose tissue leukocytosis. J Biol Chem. 2014;289(20):14045-14055.

45. Yao Y, et al. Chop deficiency protects mice against bleomycin-induced pulmonary fibrosis by attenuating M2 macrophage production. $\mathrm{Mol}$ Ther. 2016;24(5):915-925.

46. Oh J, et al. Endoplasmic reticulum stress controls M2 macrophage differentiation and foam cell formation. J Biol Chem. 2012;287(15):11629-11641.

47. Reimold AM, et al. Plasma cell differentiation requires the transcription factor XBP-1. Nature. 2001;412(6844):300-307.

48. Iwakoshi NN, Lee AH, Vallabhajosyula P, Otipoby KL, Rajewsky K, Glimcher LH. Plasma cell differentiation and the unfolded protein response intersect at the transcription factor XBP-1. Nat Immunol. 2003;4(4):321-329.

49. Brucklacher-Waldert V, et al. Cellular stress in the context of an inflammatory environment supports TGF- $\beta$-independent T helper-17 differentiation. Cell Rep. 2017;19(11):2357-2370.

50. Simonian PL, et al. Th17-polarized immune response in a murine model of hypersensitivity pneumonitis and lung fibrosis. J Immunol. 2009;182(1):657-665.

51. Yoshizaki A, et al. Cell adhesion molecules regulate fibrotic process via Th1/Th2/Th17 cell balance in a bleomycin-induced scleroderma model. JImmunol. 2010;185(4):2502-2515.

52. van Lidth de Jeude JF, et al. Induction of endoplasmic reticulum stress by deletion of Grp78 depletes Apc mutant intestinal epithelial stem cells. Oncogene. 2017;36(24):3397-3405.

53. Ghavami S, et al. Autophagy and the unfolded protein response promote pro-fibrotic effects of TGF 1 in human lung fibroblasts [published online ahead of print October 26, 2017]. Am JPhysiol Lung Cell Mol Physiol. https://doi. org/10.1152/ajplung.00372.2017.

54. Baek HA, et al. Involvement of endoplasmic reticulum stress in myofibroblastic differentiation of lung fibroblasts. Am J Respir Cell Mol Biol. 2012;46(6):731-739.

55. Matsuzaki S, et al. Physiological ER stress mediates the differentiation of fibroblasts. PLoS One. 2015;10(4):e0123578.

56. Upton JP, et al. IRE1 $\alpha$ cleaves select microRNAs during ER stress to derepress translation of proapoptotic caspase-2. Science. 2012;338(6108):818-822.

57. Heindryckx F, et al. Endoplasmic reticulum stress enhances fibrosis through IRE1 $\alpha$-mediated degradation of miR-150 and XBP-1 splicing. EMBO Mol Med. 2016;8(7):729-744.

58. Bridges JP, Wert SE, Nogee LM, Weaver TE. Expression of a human surfactant protein $\mathrm{C}$ mutation associated with interstitial lung disease disrupts lung development in transgenic mice. J Biol Chem. 2003;278(52):52739-52746.

59. Mulugeta S, Nguyen V, Russo SJ, Muniswamy M, Beers MF. A surfactant protein C precursor protein BRICHOS domain mutation causes endoplasmic reticulum stress, proteasome dysfunction, and caspase 3 activation. Am J Respir Cell Mol Biol. 2005;32(6):521-30.

60. Lawson WE, et al. Endoplasmic reticulum stress in alveolar epithelial cells is prominent in IPF: association with altered surfactant protein processing and herpesvirus infection. Am JPhysiol Lung Cell Mol Physiol. 2008;294(6):L1119-L1126.

61. Maitra M, Wang Y, Gerard RD, Mendelson CR, Garcia CK. Surfactant protein A2 mutations associated with pulmonary fibrosis lead to protein instability and endoplasmic reticulum stress. J Biol Chem. 2010;285(29):22103-22113.

62. Liu L, et al. Defective nephrin trafficking caused by missense mutations in the NPHS1 gene: insight into the mechanisms of congenital nephrotic syndrome. Hum Mol Genet. 2001;10(23):2637-2644.

63. Chen YM, Zhou Y, Go G, Marmerstein JT, Kikkawa $\mathrm{Y}$, Miner JH. Laminin beta2 gene missense mutation produces endoplasmic reticulum stress in podocytes. JAm Soc Nephrol. 2013;24(8):1223-1233.

64. Fan Q, et al. R168H and V165X mutant podocin might induce different degrees of podocyte injury via different molecular mechanisms. Genes Cells. 2009;14(9):1079-1090.

65. Lawless MW, Greene CM, Mulgrew A, Taggart CC, O'Neill SJ, McElvaney NG. Activation of endoplasmic reticulum-specific stress responses associated with the conformational disease $\mathrm{Z}$ alpha 1-antitrypsin deficiency. JImmunol. 2004;172(9):5722-5726.

66. Liberman E, et al. Activation of the grp78 and grp94 promoters by hepatitis $C$ virus E2 envelope protein. JVirol. 1999;73(5):3718-3722.

67. Tardif KD, Mori K, Siddiqui A. Hepatitis C virus subgenomic replicons induce endoplasmic reticulum stress activating an intracellular signaling pathway. J Virol. 2002;76(15):7453-7459.

68. Bueno M, et al. PINK1 deficiency impairs mitochondrial homeostasis and promotes lung fibrosis. JClin Invest. 2015;125(2):521-538.

69. Torres-Gonzalez E, et al. Role of endoplasmic reticulum stress in age-related susceptibility to lung fibrosis. Am J Respir Cell Mol Biol. 2012;46(6):748-756.

70. Dreher D, Vargas JR, Hochstrasser DF, Junod AF. Effects of oxidative stress and $\mathrm{Ca}^{2+}$ agonists on molecular chaperones in human umbilical vein endothelial cells. Electrophoresis. 1995;16(7):1205-1214

71. Macer DR, Koch GL. Identification of a set of calcium-binding proteins in reticuloplasm, the luminal content of the endoplasmic reticulum. JCell Sci. 1988;91(Pt 1):61-70.

72. Badiola N, et al. Induction of ER stress in response to oxygen-glucose deprivation of cortical cultures involves the activation of the PERK and IRE-1 pathways and of caspase-12. Cell Death Dis. 2011;2:e149.

73. Koumenis C, et al. Regulation of protein synthesis by hypoxia via activation of the endoplasmic reticulum kinase PERK and phosphorylation of the translation initiation factor eIF2alpha. Mol Cell Biol. 2002;22(21):7405-7416.

74. Price BD, Calderwood SK. Gadd 45 and Gadd 153 messenger RNA levels are increased during hypoxia and after exposure of cells to agents which elevate the levels of the glucose-regulated proteins. Cancer Res. 1992;52(13):3814-3817.

75. Price BD, Mannheim-Rodman LA, Calderwood SK. Brefeldin A, thapsigargin, and AIF4- stimulate the accumulation of GRP78 mRNA in a cycloheximide dependent manner, whilst induction by hypoxia is independent of protein synthesis. J Cell Physiol. 1992;152(3):545-552.

76. King TE Jr, Tooze JA, Schwarz MI, Brown KR, Cherniack RM. Predicting survival in idiopathic pulmonary fibrosis: scoring system and survival model. Am J Respir Crit Care Med. 2001;164(7):1171-1181.

77. Peabody JW, Peabody JW Jr, Hayes EW, Hayes EW Jr, . Idiopathic pulmonary fibrosis; its occurrence in identical twin sisters. Dis Chest. 1950;18(4):330-344.

78. Thomas AQ, et al. Heterozygosity for a surfactant protein $\mathrm{C}$ gene mutation associated with usual interstitial pneumonitis and cellular nonspecific interstitial pneumonitis in one kindred. Am J Respir Crit Care Med. 2002;165(9):1322-1328.

79. Kabore AF, Wang WJ, Russo SJ, Beers MF. Biosynthesis of surfactant protein C: characterization of aggresome formation by EGFP chimeras containing propeptide mutants lacking conserved cysteine residues. J Cell Sci. 2001;114(Pt 2):293-302.

80. Mulugeta S, Maguire JA, Newitt JL, Russo SJ, Kotorashvili A, Beers MF. Misfolded BRICHOS $\mathrm{SP}-\mathrm{C}$ mutant proteins induce apoptosis via caspase-4- and cytochrome c-related mechanisms. Am J Physiol Lung Cell Mol Physiol. 2007;293(3):L720-L729.

81. Korfei M, et al. Epithelial endoplasmic reticulum stress and apoptosis in sporadic idiopathic pulmonary fibrosis. Am J Respir Crit Care Med. 2008;178(8):838-846.

82. Korfei M, et al. Comparative proteome analysis of lung tissue from patients with idiopathic pulmonary fibrosis (IPF), non-specific interstitial pneumonia (NSIP) and organ donors. J Proteomics. 2013;85:109-128.

83. Kropski JA, et al. Extensive phenotyping of individuals at risk for familial interstitial pneumonia reveals clues to the pathogenesis of interstitial lung disease. Am J Respir Crit Care Med. 2015;191(4):417-426.

84. Geraghty P, Wallace A, D'Armiento JM. Induction of the unfolded protein response by cigarette smoke is primarily an activating transcription factor 4-C/EBP homologous protein mediated process. Int JChron Obstruct Pulmon Dis. 2011;6:309-319.

85. Jorgensen E, Stinson A, Shan L, Yang J, Gietl D, Albino AP. Cigarette smoke induces endoplasmic reticulum stress and the unfolded protein response in normal and malignant human lung cells. BMC Cancer. 2008;8:229.

86. Zhao H, Yang J, Shan L, Jorgensen ED. Measuring the impact of cigarette smoke on the UPR. Methods Enzymol. 2011;489:147-164.

87. Kamp DW, et al. Asbestos-induced alveolar epithelial cell apoptosis. The role of endoplasmic 
reticulum stress response. Am J Respir Cell Mol Biol. 2013;49(6):892-901.

88. Laing S, et al. Airborne particulate matter selectively activates endoplasmic reticulum stress response in the lung and liver tissues. $\mathrm{Am} \mathrm{J}$ Physiol Cell Physiol. 2010;299(4):C736-C749.

89. Conkright JJ, Na CL, Weaver TE. Overexpression of surfactant protein-C mature peptide causes neonatal lethality in transgenic mice. Am J Respir Cell Mol Biol. 2002;26(1):85-90.

90. Tanaka Y, et al. The exacerbating roles of CCAAT/enhancer-binding protein homologous protein (CHOP) in the development of bleomycin-induced pulmonary fibrosis and the preventive effects of tauroursodeoxycholic acid (TUDCA) against pulmonary fibrosis in mice. Pharmacol Res. 2015;99:52-56.

91. Ayaub EA, et al. GRP78 and CHOP modulate macrophage apoptosis and the development of bleomycin-induced pulmonary fibrosis. J Pathol. 2016;239(4):411-425.

92. Celardo I, et al. Mitofusin-mediated ER stress triggers neurodegeneration in pink1/parkin models of Parkinson's disease. Cell Death Dis. 2016;7(6):e2271.

93. Cybulsky AV. The intersecting roles of endoplasmic reticulum stress, ubiquitin- proteasome system, and autophagy in the pathogenesis of proteinuric kidney disease. Kidney Int. 2013;84(1):25-33.

94. Cybulsky AV. Endoplasmic reticulum stress, the unfolded protein response and autophagy in kidney diseases. Nat Rev Nephrol. 2017;13(11):681-696.

95. Fougeray S, et al. Metabolic stress promotes renal tubular inflammation by triggering the unfolded protein response. Cell Death Dis. 2011;2:e143.

96. Maekawa H, Inagi R. Stress signal network between hypoxia and ER stress in chronic kidney disease. Front Physiol. 2017;8:74.

97. Pieri M, et al. Evidence for activation of the unfolded protein response in collagen IV nephropathies. JAm Soc Nephrol. 2014;25(2):260-275.

98. Schaeffer C, Merella S, Pasqualetto E, Lazarevic D, Rampoldi L. Mutant uromodulin expression leads to altered homeostasis of the endoplasmic reticulum and activates the unfolded protein response. PLoS One. 2017;12(4):e0175970.

99. Cybulsky AV, Takano T, Papillon J, Bijian K, Guillemette J, Kennedy CR. Glomerular epithelial cell injury associated with mutant alpha-actinin-4. Am J Physiol Renal Physiol. 2009;297(4):F987-F995.

100.El Karoui K, et al. Endoplasmic reticulum stress drives proteinuria-induced kidney lesions via lipocalin 2. Nature Commun. 2016;7:10330.

101. Kemter E, Frohlich T, Arnold GJ, Wolf E, Wanke R. Mitochondrial dysregulation secondary to endoplasmic reticulum stress in autosomal dominant tubulointerstitial kidney disease - UMOD (ADTKD-UMOD). Scientific Rep. 2017;7:42970.

102. Piret SE, et al. A mouse model for inherited renal fibrosis associated with endoplasmic reticulum stress. Dis Model Mech. 2017;10(6):773-786.

103. Johnson BG, et al. Uromodulin p.Cys147Trp mutation drives kidney disease by activating ER stress and apoptosis. J Clin Invest. 2017;127(11):3954-3969.
104.Fan Y, et al. RTN1 mediates progression of kidney disease by inducing ER stress. Nat Commun. 2015;6:7841.

105. Kato M, et al. An endoplasmic reticulum stress-regulated lncRNA hosting a microRNA megacluster induces early features of diabetic nephropathy. Nature Commun. 2016;7:12864.

106.Pang XX, Bai Q, Wu F, Chen GJ, Zhang AH, Tang CS. Urotensin II induces ER stress and EMT and increase extracellular matrix production in renal tubular epithelial cell in early diabetic mice. Kidney Blood Press Res. 2016;41(4):434-449.

107. Wu J, et al. Induction of diabetes in aged C57B6 mice results in severe nephropathy: an association with oxidative stress, endoplasmic reticulum stress, and inflammation. Am J Pathol. 2010;176(5):2163-2176.

108. Hassan $\mathrm{H}$, et al. Essential role of X-box binding protein-1 during endoplasmic reticulum stress in podocytes. JAm Soc Nephrol. 2016;27(4):1055-1065.

109. Bonomo JA, et al. Association analysis of the reticulon 1 gene in end-stage kidney disease. Am J Nephrol. 2015;42(4):259-264.

110. Fan Y, et al. Inhibition of reticulon-1A-mediated endoplasmic reticulum stress in early AKI attenuates renal fibrosis development. J Am Soc Nephrol. 2017;28(7):2007-2021.

111. Fan Y, et al. Rtn1a-mediated endoplasmic reticulum stress in podocyte injury and diabetic nephropathy. Scientific Rep. 2017;7(1):323.

112. Xiao W, Fan Y, Wang N, Chuang PY, Lee K, He JC. Knockdown of RTN1A attenuates ER stress and kidney injury in albumin overloadinduced nephropathy. Am J Physiol Renal Physiol. 2016;310(5):F409-F415.

113. Ke PY, Chen SS. Hepatitis C virus and cellular stress response: implications to molecular pathogenesis of liver diseases. Viruses. 2012;4(10):2251-2290.

114. Baiceanu A, Mesdom P, Lagouge M, Foufelle F Endoplasmic reticulum proteostasis in hepatic steatosis. Nat Rev Endocrinol. 2016;12(12):710-722.

115. Sasaki M, Yoshimura-Miyakoshi M, Sato Y, Nakanuma Y. A possible involvement of endoplasmic reticulum stress in biliary epithelial autophagy and senescence in primary biliary cirrhosis. J Gastroenterol. 2015;50(9):984-995.

116. Hidvegi T, et al. An autophagy-enhancing drug promotes degradation of mutant alpha1antitrypsin $\mathrm{Z}$ and reduces hepatic fibrosis. Science. 2010;329(5988):229-232.

117. Teckman JH, Perlmutter DH. Retention of mutant alpha(1)-antitrypsin $\mathrm{Z}$ in endoplasmic reticulum is associated with an autophagic response. Am J Physiol Gastrointest Liver Physiol. 2000;279(5):G961-G974.

118. Kroeger H, et al. Endoplasmic reticulumassociated degradation (ERAD) and autophagy cooperate to degrade polymerogenic mutant serpins. J Biol Chem. 2009;284(34):22793-22802.

119. Li X, Wang Y, Wang H, Huang C, Huang Y, Li J. Endoplasmic reticulum stress is the crossroads of autophagy, inflammation, and apoptosis signaling pathways and participates in liver fibrosis. Inflamm Res. 2015;64(1):1-7.

120. Maiers JL, et al. The unfolded protein response mediates fibrogenesis and collagen I secretion through regulating TANGO1 in mice. Hepatology.
2017;65(3):983-998.

121. Koo JH, Lee HJ, Kim W, Kim SG. Endoplasmic reticulum stress in hepatic stellate cells promotes liver fibrosis via PERK-mediated degradation of HNRNPA1 and up-regulation of SMAD2. Gastroenterology. 2016;150(1):181-193.e8.

122. Jiang $\mathrm{S}$, et al. Fibroblast growth factor 21 is regulated by the IRE1alpha-XBP1 branch of the unfolded protein response and counteracts endoplasmic reticulum stress-induced hepatic steatosis. J Biol Chem. 2014;289(43):29751-29765.

123. Kim SH, et al. Fibroblast growth factor 21 participates in adaptation to endoplasmic reticulum stress and attenuates obesity-induced hepatic metabolic stress. Diabetologia. 2015;58(4):809-818.

124.Schaap FG, Kremer AE, Lamers WH, Jansen PL, Gaemers IC. Fibroblast growth factor 21 is induced by endoplasmic reticulum stress. Biochimie. 2013;95(4):692-699.

125. Wan XS, et al. ATF4- and CHOP-dependent induction of FGF21 through endoplasmic reticulum stress. Biomed Res Int. 2014;2014:807874.

126. Cho EJ, et al. Tauroursodeoxycholic acid attenuates progression of steatohepatitis in mice fed a methionine-choline-deficient diet. Dig Dis Sci. 2014;59(7):1461-1474.

127. Henkel AS, Dewey AM, Anderson KA, Olivares S, Green RM. Reducing endoplasmic reticulum stress does not improve steatohepatitis in mice fed a methionine- and choline-deficient diet. Am J Physiol Gastrointest Liver Physiol. 2012;303(1):G54-G59.

128. Tamaki N, et al. CHOP deficiency attenuates cholestasis-induced liver fibrosis by reduction of hepatocyte injury. Am J Physiol Gastrointest Liver Physiol. 2008;294(2):G498-G505.

129. Gu X, et al. Bip overexpression, but not CHOP inhibition, attenuates fatty-acid-induced endoplasmic reticulum stress and apoptosis in HepG2 liver cells. Life Sci. 2010;87(23-26):724-732.

130. Pfaffenbach KT, Gentile CL, Nivala AM, Wang D, Wei Y, Pagliassotti MJ. Linking endoplasmic reticulum stress to cell death in hepatocytes: roles of $\mathrm{C} / \mathrm{EBP}$ homologous protein and chemical chaperones in palmitate-mediated cell death. Am J Physiol Endocrinol Metab. 2010;298(5):E1027-E1035.

131. Yi HW, et al. Ethanol promotes saturated fatty acid-induced hepatoxicity through endoplasmic reticulum (ER) stress response. Chin J Nat Med. 2015;13(4):250-256.

132. Kaser A, et al. XBP1 links ER stress to intestinal inflammation and confers genetic risk for human inflammatory bowel disease. Cell. 2008;134(5):743-756

133. Heazlewood CK, et al. Aberrant mucin assembly in mice causes endoplasmic reticulum stress and spontaneous inflammation resembling ulcerative colitis. PLoS Med. 2008;5(3):e54.

134. Ayala $P$, et al. Attenuation of endoplasmic reticulum stress using the chemical chaperone 4phenylbutyric acid prevents cardiac fibrosis induced by isoproterenol. Exp Mol Pathol. 2012;92(1):97-104.

135. Kassan M, et al. Endoplasmic reticulum stress is involved in cardiac damage and vascular endothelial dysfunction in hypertensive mice. Arterioscler Thromb Vasc Biol. 2012;32(7):1652-1661. 
136. Choi SW, Ryu OH, Choi SJ, Song IS, Bleyer AJ, Hart TC. Mutant tamm-horsfall glycoprotein accumulation in endoplasmic reticulum induces apoptosis reversed by colchicine and sodium 4-phenylbutyrate. J Am Soc Nephrol. 2005;16(10):3006-3014.

137. Liu SH, et al. Chemical chaperon 4phenylbutyrate protects against the endoplasmic reticulum stress-mediated renal fibrosis in vivo and in vitro. Oncotarget. 2016;7(16):22116-22127.

138. Wang JQ, et al. Phenylbutyric acid protects against carbon tetrachloride-induced hepatic fibrogenesis in mice. Toxicol Appl Pharmacol. 2013;266(2):307-316.

139. Zhao H, et al. Phenylbutyric acid inhibits epithelial-mesenchymal transition during bleomycin-induced lung fibrosis. Toxicol Lett. 2015;232(1):213-220.

140.Zhou W, Bercury K, Cummiskey J, Luong N, Lebin J, Freed CR. Phenylbutyrate up-regulates the DJ-1 protein and protects neurons in cell culture and in animal models of Parkinson disease. J Biol Chem. 2011;286(17):14941-14951.

141. Ghosh R, et al. Allosteric inhibition of the IRE1alpha RNase preserves cell viability and function during endoplasmic reticulum stress. Cell. 2014;158(3):534-548.

142.Imanikia S, Sheng M, Taylor RC. Cell Nonautonomous UPRER signaling [published online ahead of print September 7, 2017]. Curr Top Microbiol Immunol. https://doi. org/10.1007/82_2017_38.
143. Ribeiro CM, et al. Chronic airway infection/inflammation induces a $\mathrm{Ca}^{2+}{ }_{i}$-dependent hyperinflammatory response in human cystic fibrosis airway epithelia. J Biol Chem. 2005;280(18):17798-17806.

144. Mahadevan NR, Rodvold J, Sepulveda H, Rossi S, Drew AF, Zanetti M. Transmission of endoplasmic reticulum stress and pro-inflammation from tumor cells to myeloid cells. Proc Natl Acad Sci US A. 2011;108(16):6561-6566.

145. Taylor RC, Dillin A. XBP-1 is a cellnonautonomous regulator of stress resistance and longevity. Cell. 2013;153(7):1435-1447.

146. Kanemoto S, et al. Multivesicular body formation enhancement and exosome release during endoplasmic reticulum stress. Biochem Biophys Res Commun. 2016;480(2):166-172. 